\title{
Changes in Cardiac Autonomic Modulation in Women with Breast Cancer Using Aromatase Inhibitors and the Relation with Biochemical Variables
}

\author{
Luana Almeida Gonzaga, ${ }^{1 \oplus}$ Thais Reis Silva de Paulo, ${ }^{2}{ }^{\circledR}$ Juliana Viezel, ${ }^{\circledR}{ }^{\circledR}$ Laís Manata Vanzella, ${ }^{1 \oplus}$ Ismael Forte \\ Freitas Jr., ${ }^{3 \oplus}$ Luiz Carlos Marques Vanderlei ${ }^{1 \oplus}$ \\ Departamento de Fisioterapia - Faculdade de Ciências e Tecnologia da Universidade Estadual Paulista (UNESP), ${ }^{1}$ Presidente Prudente, SP - Brazil \\ Departamento de Educação Física - Universidade Federal do Rio Grande do Norte (UFRN), ${ }^{2} \mathrm{Natal}, \mathrm{RN}-\mathrm{Brazil}$ \\ Departamento de Educação Física - Faculdade de Ciências e Tecnologia da Universidade Estadual Paulista (UNESP), ${ }^{3}$ Presidente Prudente, SP - Brazil
}

\begin{abstract}
Background: The use of autonomic modulation as a predictor of cardiovascular risk in women with breast cancer is important.

Objective: To evaluate the cardiac autonomic modulation of postmenopausal women using aromatase inhibitors for breast cancer treatment, as well as its relation with the following biochemical variables.

Methods: Postmenopausal women who did not have breast cancer $(n=33)$ and postmenopausal women with breast cancer $(n=15)$. For evaluation of the autonomic modulation the heart rate was recorded beat-to-beat for 30 minutes and the series of RR intervals obtained were used to calculate the following heart rate variability indices: Mean RR ms, SDNN (standard deviation of all normal RR intervals, expressed in milliseconds) ms, Mean HR, RMSSD (square root of the mean of the squared differences between adjacent normal RR interval) ms, NN50 (number of pairs of successive NNs that differ by more than $50 \mathrm{~ms}$ ) count, pNN50\% (proportion of NN50 divided by total number of NNs), RRtri (RR triangular), TINN (triangular interpolation of NN interval) $\mathrm{ms}$, SD1 ms, SD2 ms, LF $\mathrm{ms}^{2}$, HF $\mathrm{ms}^{2}$, LH/HF $\mathrm{ms}^{2}$. The values of biochemical variables (fasting glycemia, triglycerides, HDL-cholesterol, and C-reactive protein) were analyzed by blood sample.
\end{abstract}

Results: Lower values of heart rate variability indices were observed in postmenopausal women with breast cancer in relation to postmenopausal women who did not have breast cancer: Mean RR $(p=0.03)$; $\operatorname{sDNN}(p=0.03)$; $R$ MSSD $(p=0.03) ;$ NN50 count $(p=0.03) ;$ pNN50 \% $(p=0.03) ;$ RRtri $(p=0.02) ;$ SD1 $(p=0.01) ;$ SD2 $(p=0.02) ;$ LF ms ${ }^{2}$ $(p=0.01) ; \mathrm{HF} \mathrm{ms}^{2}(p=0.03)$. There was an inversely proportional correlation between the indices SDNN, SD2, and $\mathrm{HFms}^{2}$ with triglycerides (SDNN $\mathrm{p}=0.04 ; \mathrm{SD} 2 \mathrm{p}=0.04 ; \mathrm{HF} \mathrm{ms}^{2} \mathrm{p}=0.04$ ). No statistically significant correlations were found between heart rate variability indices and others variables. Statistical significance was set at $5 \%$ for all analyses.

Conclusion: Women with breast cancer present reduced autonomic modulation and in these women of heart rate variability indices are inversely correlated with triglyceride values. (Arq Bras Cardiol. 2019; 112(5):555-563)

Keywords: Aromatase Inhibitors; Breast Neoplasms; Cholesterol; Blood Glucose; Cardiovascular Dieases/prevention and control; Estrogen Replacement Therapy/adverse effects.

\section{Introduction}

Breast cancer is the second most common type of neoplasm in the world and the most common among women. Annually, in both developing and developed countries, about $22 \%$ of new cases arise ${ }^{1}$ and, according to the World Health Organization, ${ }^{2}$ in 2011 more than 508,000 deaths occurred worldwide due to this disease.

Chemotherapy, radiation therapy, and hormone therapy can be used as treatment for breast cancer. These treatments

Mailing Address: Thais Reis Silva de Paulo •

Campus Universitário UFRN - Centro de Ciência da Saúde - Departamento de Educação Física. Postal Code 59078-970, Lagoa Nova, Natal, RN - Brazil Email: thais.reis.silva@hotmail.com

Manuscript received March 27, 2018, revised manuscript August 06, 2018, accepted August 15, 2018

DOI: $10.5935 / a b c .20190036$ are indispensable and promote positive impacts on the cure for cancer, recurrence, and metastasis; however, the side effects can cause numerous discomforts, compromising other aspects related to women's health. ${ }^{3}$

Among the side effects precipitated by the various treatments for breast cancer, those related to hormone therapy, more specifically to the use of aromatase inhibitors (AI), deserve attention. These substances block the enzyme aromatase, responsible for the conversion of androgens to estrogens in postmenopausal women. ${ }^{3-5}$

Estrogen is associated with a better lipid profile and an increase in the synthesis of vasodilatory enzymes, ${ }^{6,7}$ so its reduction in women with breast cancer promoted by the use of $\mathrm{Al}$, associated with its lower production in the menopausal period, may be related to a worse lipid profile and, consequently, a higher risk of cardiovascular diseases (CVD). ${ }^{6,7}$

In this context, the evaluation of women with breast cancer who are in the menopause becomes fundamental so that CVD 
can be avoided and/or prevented. Autonomic modulation analysis can be used for this purpose since the autonomic nervous system (ANS) is one of the components involved in the etiology and consequences of cardiovascular disorders caused by the treatment of breast cancer. ${ }^{8,9}$

The use of autonomic modulation as a predictor of cardiovascular risk in women with breast cancer was evidenced by Lakoski et al., ${ }^{9}$ who identified autonomic dysfunctions in these women characterized by increased sympathetic modulation and decreased parasympathetic modulation, suggesting a higher risk of CVD in women with breast cancer.

As can be observed, the risk of CVD in women with menopausal breast cancer may be related to a reduction in autonomic modulation and worsening of the lipid profile, which can be precipitated by both menopause and the use of $\mathrm{Al} .{ }^{10,11}$

These women are also more prone to weight gain after chemotherapy and, consequently, to suffer changes in visceral adiposity, leading to changes in lipid profile and insulin resistance. ${ }^{3}$ In addition, elevated levels of inflammation have been observed in cancer patients, ${ }^{12}$ a condition also responsible for lower survival in these patients. ${ }^{12,13}$

However, the correlation between autonomic modulation and these factors has not been explored. Therefore, investigating the autonomic modulation of women with breast cancer in menopause who use $\mathrm{Al}$ and the relationship with cardiovascular biochemical variables could improve the targeting of future treatments and quality of life of women with breast cancer.

In this context, this study aimed to evaluate the cardiac autonomic modulation of postmenopausal women using AI to treat breast cancer, as well as its relationship with the following cardiovascular biochemical variables: fasting glycemia, triglycerides, HDL cholesterol, and C-reactive protein (CRP).

\section{Methods}

This is a cross-sectional study, carried out from March 2015 to July 2016, in a city in the southeastern region of Brazil. A total of 348 women, who were treated for breast cancer and registered in the records of the Oncology Pharmacy of the city's Regional Hospital, were analyzed. The medical records of these patients were analyzed and only women who were using Al were invited to participate in the study, totalling 124 women. Postmenopausal women without breast cancer were invited and recruited through radio, television, and local newspapers, totalling 189 women.

The inclusion criteria of the study were: aged between 50 and 80 years; being in the menopause, defined by the self report of absence of the menstrual cycle in the previous 12 months; signing the informed consent form to participate in the study, and not having participated in supervised physical exercise for at least six months immediately prior to the study. Specifically for women with breast cancer, in addition to all the criteria mentioned above, they were required to present stages I to IIla of breast cancer, ${ }^{14}$ certified by doctors through the medical records.

The study was approved by the Institution's Ethics and Research Committee (Protocol No. 6727715.1.0000.5402/2015) and registered in the ClinicalTrials.gov Platform with the identifier NCT02804308.

\section{Experimental draw}

The experimental design of the present study included two groups of women with different characteristics: one with and one without breast cancer. According to the inclusion criteria specific to this study, the convenience sampling consisted of 48 postmenopausal women, who were distributed as follows: 33 without breast cancer and 14 survivors of breast cancer under treatment with Al. The selection of the participants in this study can be better visualized in figure 1 .

On the first day, all the women participating in the study answered questionnaires related to sociodemographic information (with questions related to age, education level, marital status, occupation, children, and self-reported diseases - cardiac, respiratory, metabolic, musculoskeletal). After answering the questionnaires, the volunteers underwent an evaluation of body composition using DEXA equipment - Dual Energy X-ray Absorptiometry, brand Lunar DPX-NT. Subsequently, the volunteers received a referral to the clinical analysis laboratory for blood sample collections, heart rate variability (HRV) assessments were scheduled, and the guidelines provided.

For HRV analysis, the heart rate was recorded beat-to-beat in the morning ( $8 \mathrm{am}$ to $11 \mathrm{am}$ ) in a quiet environment with a temperature between $21^{\circ} \mathrm{C}$ and $24^{\circ} \mathrm{C}$ and relative air humidity of $40-60 \%$ and the series of RR intervals obtained were used for the calculation of HRV indices.

\section{Body composition}

Body composition was measured using DEXA Dual Energy X-ray Absorptiometry, brand Lunar DPX-NT, General Electric Healthcare, Little Chalfont, Buckinghamshire, UK, software version 4.7. The following outcome variables were collected: percentage of body fat $(\%)$, lean mass $(\mathrm{kg})$, fat mass $(\mathrm{kg})$, and total bone mineral density $\left(\mathrm{g} / \mathrm{cm}^{2}\right)$.

\section{Analysis of HRV}

For the analysis of HRV, initially, the volunteers were instructed not to consume alcoholic beverages and/or ANS stimulants such as coffee, tea, soda, and chocolate, and not to perform any type of intense physical exercise during the 24 hours preceding the evaluation.

The Polar S810i heart rate monitor (Polar, Finland), previously validated equipment for recording heart rate and its use for calculation of $\mathrm{HRV}^{15}$ indices, was used to record heart rate. The equipment pick-up strap was positioned on the distal third of the sternum and the heart rate receiver on the volunteer's wrist. During the uptake, the volunteers were instructed to remain silent, awake, at rest, and breathing spontaneously for 30 minutes in the supine position.

For the analysis of the HRV indices, 1000 RR intervals were obtained from the most stable section of the trace, which was subjected to digital filtering in the proprietary software of the cardio-frequency meter, Polar Pro Trainer 5 version 5.41.002, complemented by manual filtering for elimination 


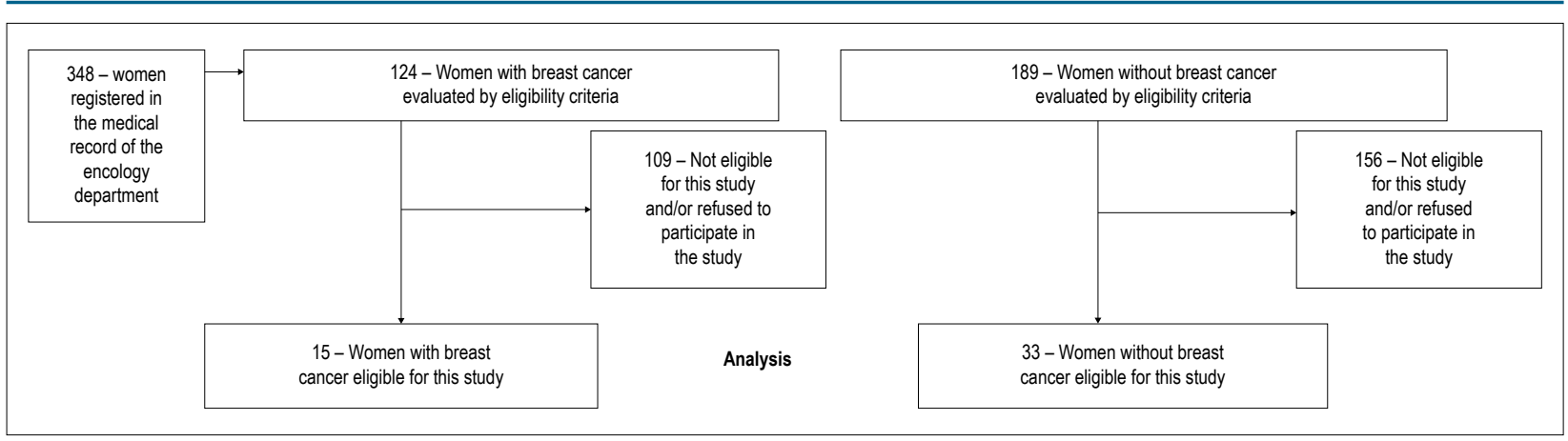

Figure 1 - Study design: recruitment and analysis.

of premature ectopic beats and artefacts and only series of RR intervals that presented more than $95 \%$ of sinus beats were included in the study. Calculations of the HRV indices were performed using Kubios HRV Analysis software version 2.0 (Kuopio University, Finland). ${ }^{16}$

The following indices were analyzed in the time domain: Mean RR, which represents the mean RR intervals; Mean $H R$, which corresponds to mean heart rate; SDNN, which represents the standard deviation of all normal RR intervals; RMSSD, which corresponds to the square root of the sum of the square of the differences between the RR intervals in the record, divided by the number of RR intervals in a given time minus one RR interval; and finally the NN50 (number of pairs of successive NNs that differ by more than $50 \mathrm{~ms}$ ) and pNN50 (proportion of NN50 divided by total number of NNs), where the NN50 is the counter of the number of times that successive $\mathrm{NN}$ intervals present a duration difference greater than $50 \mathrm{~ms}$, and the pNN50 is the ratio obtained by the NN50 / $\mathrm{n}$ ratio. ${ }^{17,18}$

In the frequency domain, low frequency (LF: $0.04-0.15 \mathrm{~Hz}$ ) and high frequency (HF: $0.15-0.40 \mathrm{~Hz}$ ) spectral components were analyzed in $\mathrm{ms}^{2}$ as well as the ratio between the components (LF/HF). The spectral analysis was calculated using the Fast Fourier Transform algorithm. ${ }^{18}$

In addition, the RRtri (RR triangular), TINN (triangular interpolation of $\mathrm{NN}$ interval), and Poincaré plot were also calculated, which was quantitatively and qualitatively analyzed. The RRtri and TINN were calculated by constructing a density histogram of normal RR intervals, which contains the length of the RR intervals on the $x$-axis and the frequency with which they occurred on the $y$-axis. ${ }^{18}$ The union of the points of the columns of the histogram forms a figure similar to a triangle from which these indices are extracted. ${ }^{18}$

The Poincaré plot is the two-dimensional graphical representation of the correlation between consecutive RR intervals, where each interval is plotted against the next interval. For quantitative analysis of the plot, the SD1 (standard deviation of instantaneous beat-to-beat variability) and SD2 indices (long-term standard deviation of continuous RR intervals) were calculated. ${ }^{18}$

The qualitative analysis of the plot was carried out through the analysis of the figures formed by its attractor, which demonstrates the degree of complexity of the RR intervals.
The following patterns were considered: I) Figure showing an increase in the dispersion of RR intervals, characteristic of a normal plot; II) Figure with small global dispersion, with no increase in the dispersion of RR intervals in the long term, characteristic of a plot with lower variability. ${ }^{19}$

\section{Blood samples}

Blood collection was performed in a private laboratory and for the biochemical analyzes the volunteers respected a 12-hour fast. The collection was performed in a vacuum tube with separator gel without anticoagulant; after collection, the blood was centrifuged for 10 minutes at $3000 \mathrm{rpm}$ to separate the serum from the other blood components, which was used for the analyzes.

For the determination of glycemia, triglycerides, and the HDL-cholesterol fraction, a colourimetric enzyme kit was used in an Autohumalyzer A517 device ${ }^{17}$ (HUMAN et al., 2004). CRP was measured using an enzyme ELISA kit: Immulite 2000 analyzer (Siemens Healthcare Diagnostics). ${ }^{20}$

\section{Statistical analysis}

The descriptive data for characterization of the sample are expressed as percentage, mean, standard deviation. In order to compare the anthropometric variables, age, and the HRV indices between the groups, the normality of the data was initially tested using the Shapiro-Wilk test. If the normal distribution was accepted, the Student's t-test for unpaired data was applied, while for non-normal distributions the Mann-Whitney test was applied. The continuous variables that did not present normal distribution were described through median and interquartile range and those that presented normal distribution were described through mean and standard deviation.

The correlation between the HRV indices and the independent variables CRP, fasting glycemia, triglycerides, and HDL-cholesterol was verified by linear regression in unadjusted and adjusted models, considering the age of the volunteers.

For both analyzes, statistically, significant differences were considered when the " $p$ " value was lower than 0.05 . The program used for statistical analysis was the "Statistical Package for Social Sciences" version 15.0 (SPSS Inc., Chicago, Illinois, United States of America). 


\section{Results}

Table 1 presents the sociodemographic and clinical characteristics of the postmenopausal women without breast cancer and breast cancer survivors who participated in the study. There were no significant differences between groups ( $p>0.05)$. The losses of the sample composition can be visualized in Figure 1.

Approximately $70.8 \%$ of women who survived breast cancer and $25 \%$ of the non-cancer group attended high school. Regarding marital status, $70.8 \%$ of women who survived and $60.1 \%$ without the disease are married. Of the women analyzed, $41.7 \%$ of the survivors of the disease and $55.6 \%$ of those without the disease worked from the home, while, $62.4 \%$ and $72.8 \%$, respectively, reported having up to two diseases. Regarding the body composition variables analyzed (total body mass, BMI, total lean and fat mass, trunk fat mass, bone mineral density, and bone mineral content), there were no statistically significant differences between the groups.

In women with breast cancer, it was observed that 52\% had undergone mastectomy surgery and $67 \%$ received treatment with chemotherapy. Most women have diagnosed in breast cancer stage I and the mean time of Al use was 19.3 months.

The Mean RR, SDNN, RMSSD, NN50, pNN50, RRtri, SD1, and SD2 indices demonstrated statistically significant reductions (Table 2$)(p \leq 0.05)$ in the breast cancer group when compared to the group without the disease. The Mean HR index (1/me), which represents the heart rate $(\mathrm{HR})$, was lower in the group without the disease. For the TINN index, no significant differences were found between groups ( $p=0.216)$.

In the qualitative analysis of the Poincaré plot, there was a lower dispersion of the RR intervals in the cancer group compared to the non-disease group (Figure 2).

The indices in the frequency domain of HRV are presented in table 3. For the VLF $\left(\mathrm{ms}^{2}\right)$, LF $\left(\mathrm{ms}^{2}\right)$, and $\mathrm{HF}\left(\mathrm{ms}^{2}\right)$ indices, statistically significant reductions $(p \leq 0.05)$ were found in the breast cancer group when compared to the group without the disease, whereas the LF/HF ratio did not present significant differences between the groups $(p=0.747)$.

Table 1 - Sociodemographic and clinical characteristics of postmenopausal women without breast cancer and breast cancer survivors who participated in the study

\begin{tabular}{|c|c|c|c|}
\hline Variables & Group with Breast Cancer $(n=14)$ & Group without Breast Cancer $(n=27)$ & $p$ value \\
\hline $\mathrm{Age}^{\mathrm{a}}$ & $62.17 \pm 5.79$ & $60.03 \pm 7.57$ & 0.23 \\
\hline \multicolumn{4}{|l|}{ Education } \\
\hline Illiterate ${ }^{\mathrm{b}}$ & $8.3 \%$ & $12.4 \%$ & \\
\hline First level completed ${ }^{b}$ & $16.7 \%$ & $37.6 \%$ & 0.63 \\
\hline Second level Completed ${ }^{b}$ & $70.8 \%$ & $25 \%$ & \\
\hline Superior ${ }^{b}$ & $4.2 \%$ & $25 \%$ & \\
\hline \multicolumn{4}{|l|}{ Marital status } \\
\hline Single ${ }^{b}$ & $4.2 \%$ & $11.1 \%$ & \\
\hline Married $^{b}$ & $70.8 \%$ & $61.1 \%$ & 0.49 \\
\hline Divorced ${ }^{b}$ & $16.7 \%$ & $5.6 \%$ & \\
\hline Widow $^{b}$ & $8.3 \%$ & $22.2 \%$ & \\
\hline \multicolumn{4}{|l|}{ Occupation } \\
\hline From home $^{b}$ & $41.7 \%$ & $55.6 \%$ & \\
\hline Work $^{b}$ & $41.7 \%$ & $5.6 \%$ & 0.25 \\
\hline Retired $^{b}$ & $16.6 \%$ & $38.8 \%$ & \\
\hline \multicolumn{4}{|l|}{ Self-Reported diseases } \\
\hline Up to 2 diseases ${ }^{b}$ & $62.4 \%$ & $72.8 \%$ & 0.49 \\
\hline More than 2 diseases ${ }^{b}$ & $16.6 \%$ & $27.8 \%$ & \\
\hline Total body mass $(\mathrm{kg})^{\mathrm{a}}$ & $70.62 \pm 12.29$ & $69.67 \pm 12.99$ & 0.61 \\
\hline $\mathrm{BM}^{\mathrm{a}}$ & $30.71 \pm 6.03$ & $30.19 \pm 4.57$ & 0.59 \\
\hline Total lean mass $(\mathrm{kg})^{\mathrm{a}}$ & $34.94 \pm 4.39$ & $35.53 \pm 4.76$ & 0.19 \\
\hline Total fat mass $(\mathrm{kg})^{\mathrm{a}}$ & $33.38 \pm 9.53$ & $31.83 \pm 9.54$ & 0.36 \\
\hline Fat mass of thoracic $(\mathrm{kg})^{\mathrm{a}}$ & $18.17 \pm 4.84$ & $16.70 \pm 4.75$ & 0.24 \\
\hline $\mathrm{BMD}^{\mathrm{a}}$ & $1.11 \pm 0.11$ & $1.17 \pm 0.12$ & 0.09 \\
\hline $\mathrm{BMC}^{\mathrm{a}}$ & $2.12 \pm 0.32$ & $2.31 \pm 0.32$ & 0.11 \\
\hline
\end{tabular}

Student test: Mean \pm standard deviation; Qui Square test': categorical variables, percentage. BMl: body mass index; BMD: bone mineral density; BMC: bone mineral content. 


\section{Original Article}

Table 2 - Linear indices in time domain of heart rate variability in each group

\begin{tabular}{|c|c|c|c|}
\hline & Group with breast cancer $(n=14)$ & Group without breast cancer $(n=27)$ & $p$ value \\
\hline Mean $\mathrm{RR}_{\mathrm{ms}}^{\mathrm{a}}$ & $707.76 \pm 89.86$ & $867.80 \pm 128.87$ & 0.047 \\
\hline $\mathrm{SDNN} \mathrm{ms}^{\mathrm{a}}$ & $17.92 \pm 5.05$ & $35.20 \pm 14.94$ & 0,042 \\
\hline Mean HR $1 / \mathrm{mim}^{\mathrm{a}}$ & $85.89 \pm 10.49$ & $70.77 \pm 10.75$ & 0,050 \\
\hline RMSSD $\mathrm{ms}^{\mathrm{b}}$ & $11.30 \pm 8.40$ & $18.40 \pm 16.00$ & 0.010 \\
\hline NN50 count ${ }^{b}$ & $0.00 \pm 4.00$ & $15.00 \pm 90.50$ & 0.022 \\
\hline pNN50 \% ${ }^{b}$ & $0.00 \pm 0,40$ & $1.50 \pm 9.05$ & 0.022 \\
\hline RRtria & $5.76 \pm 1.58$ & $9.93 \pm 4.01$ & 0.015 \\
\hline TINN $\mathrm{ms}^{\mathrm{a}}$ & $86.00 \pm 22.19$ & $138.40 \pm 79.38$ & 0.243 \\
\hline $\mathrm{SD} 1 \mathrm{~ms}^{\mathrm{b}}$ & $8.00 \pm 5.75$ & $13.00 \pm 11.35$ & 0.009 \\
\hline $\mathrm{SD} 2 \mathrm{~ms}^{\mathrm{a}}$ & $23.90 \pm 6.51$ & $46.91 \pm 19.95$ & 0.022 \\
\hline
\end{tabular}

Student t testa: Mean \pm standard deviation; Mann Whitney testb: Median \pm interquartile range; SDNN: standard deviation of all normal RR intervals; HR: heart rate; RMSSD: square root of the mean of the squared differences between adjacent normal RR interval; NN50: number of pairs of successive NNs that differ by more than 50 ms; pNN50: proportion of NN50 divided by total number of NNs; RRtri: RR triangular; TINN: triangular interpolation of NN interval; SD1: instantaneous variability of beat-to-beat variability, SD2: long-term standard deviation of continuous RR intervals index; ms: milliseconds.

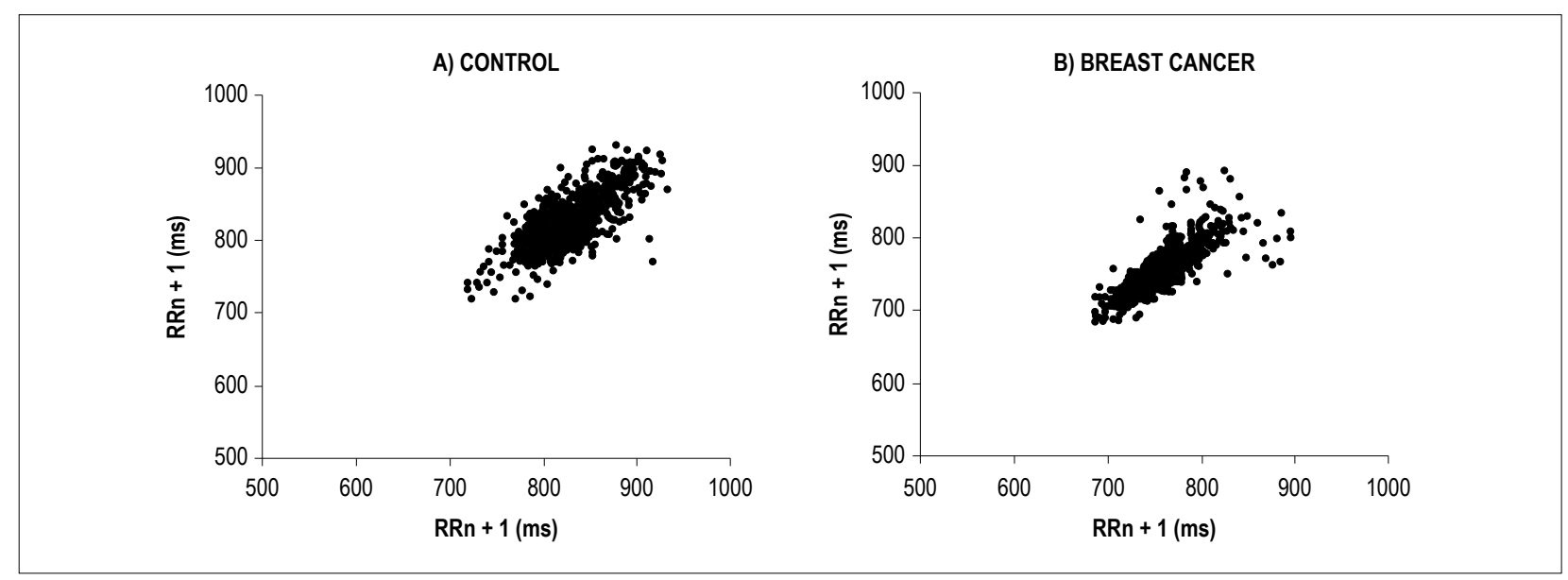

Figure 2 - Qualitative analysis of the Poincare plot. SD1 (standard deviation of instantaneous variability), SD2 (standard deviation of the continuous RR intervals). Control: $S D 1=18 \mathrm{~ms} ;$ SD2 $=45 \mathrm{~ms} /$ Breast Cancer: $S D 1=10.9 \mathrm{~ms} ; \mathrm{SD2}=32.6 \mathrm{~ms}$.

Table 4 shows the correlation of HRV indices with the inflammatory marker PCR and table 5 presents the variables blood glucose, triglycerides, and high-density lipoprotein with and without age adjustments in women with breast cancer.

There were no statistically significant correlations between the indices and the inflammatory marker, even when adjusted for age $(p>0.05)$, or between the Mean RR, Mean HR, RMSSD, NN50, pNN50, RR tri, TINN, VLF $\mathrm{ms}^{2}$, LF $\mathrm{ms}^{2}$, $\mathrm{LF} / \mathrm{HF}$, and SD1 for the variables blood glucose, triglycerides, and high density lipoprotein $(\mathrm{p}>0.05)$.

When adjusted for age the RR tri, HF $\mathrm{ms}^{2}$, and SD2 indices presented inversely proportional correlations with the triglycerides [HF - $\beta 95 \% \mathrm{Cl}=-0.53, p=0.045 ; \mathrm{SD} 2-\beta 95 \%$ $\mathrm{Cl}=-0.13, \mathrm{p}=0.044]$; RRtri $-\beta 95 \% \mathrm{Cl}=-0.02, \mathrm{p}=0.046]$.

\section{Discussion}

The main findings of this study demonstrate that women who are survivors of breast cancer who use Al present reductions in HRV compared to women without cancer and that these HRV indices correlate with the lipid profile.

In the present study, a statistically significant reduction in the SDNN and RR tri indices, as well as the RMSSD, SD1, NN50, and HF indices, can be observed in women with breast cancer using $\mathrm{Al}$ when compared to the non-cancer group, suggesting a reduction in global and vagal modulation, respectively. Moreover, the visual analysis of the Poincaré plot demonstrates a lower dispersion of the RR intervals in the cancer group, indicating that these women present HRV reduction. 
Table 3 - Linear indices in frequency domain of heart rate variability in each group

\begin{tabular}{lccc}
\hline & Group with breast cancer $(n=14)$ & Group without breast cancer $(n=27)$ & $p$ value \\
\hline $\mathrm{LF} \mathrm{ms}^{2 b}$ & $67.00 \pm 46.50$ & $203.00 \pm 257.50$ & 0.009 \\
$\mathrm{HF} \mathrm{ms}{ }^{2 b}$ & $70.00 \pm 61.50$ & $136.00 \pm 264.00$ & 0.008 \\
$\mathrm{LF} / \mathrm{HF} \mathrm{ms}{ }^{2 b}$ & $0.47 \pm 0.99$ & $0.71 \pm 0.66$ & 0.564 \\
\hline
\end{tabular}

Mann Whitney testb: Median \pm interquartile range; $m s^{2}$ : milliseconds; LF: low frequency; HF: high frequency; VLF: very low frequency.

Table 4 - Correlation of heart rate variability indices with the inflammatory marker PCR with and without adjustments for age in woman with breast cancer

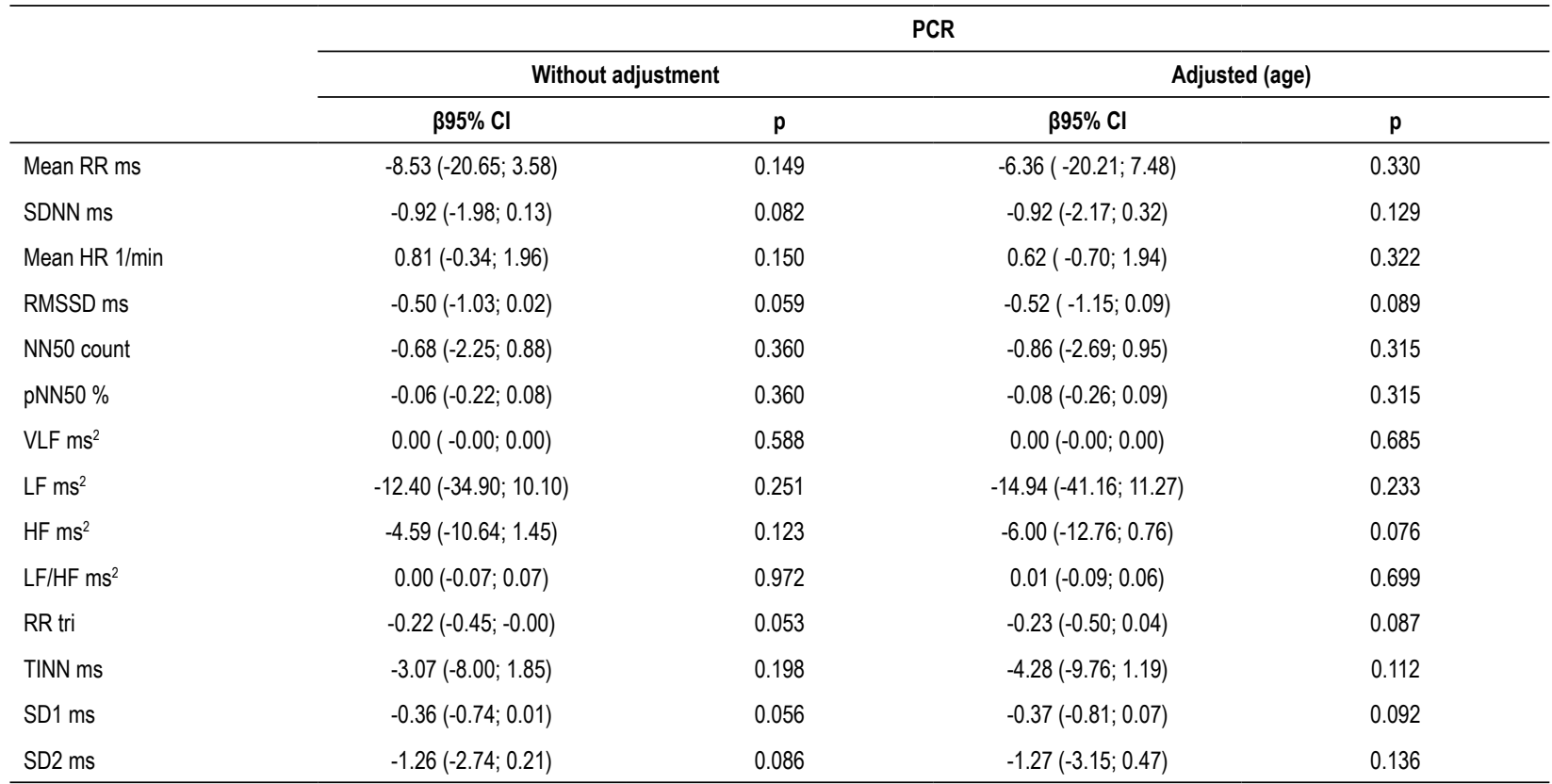

PCR: C reactive protein; SDNN: standard deviation of all normal RR intervals, expressed in milliseconds; HR: heart rate; RMSSD: square root of the mean of the squared differences between adjacent normal RR intervals; NN50: number of pairs of successive NNs that differ by more than $50 \mathrm{~ms}$; pNN50: proportion of NN50 divided by total number of NNs; TINN: triangular interpolation of NN interval; VLF: very low frequency; LF: low frequency; HF: high frequency; RR tri: RR triangular; SD1: instantaneous variability of beat-to-beat variability; SD2: long-term standard deviation of continuous RR intervals index; ms: milliseconds.

Reduction in global variability and parasympathetic modulation was also observed by Caro-Morán et al. ${ }^{8}$ in women with breast cancer undergoing chemotherapy, radiotherapy, and hormone therapy after 1 year of treatment. The authors observed reduced values of SDNN, RMSSD, and $\mathrm{HF} \mathrm{ms}^{2}$ in the cancer group in relation to the group without the disease. In these women, no significant differences were found in the $\mathrm{LF} \mathrm{ms}^{2}$ and LF/HF indices, although higher values were found in the cancer group.

It is important to note that higher parasympathetic modulation has been reported as an important factor related to a better prognosis for these patients, as observed by Giese-Davis et al. ${ }^{21}$ who reported that high baseline $\mathrm{HF}$ values were associated with higher survival and lower cardiovascular risk. Greater parasympathetic modulation is generally associated with lower cardiovascular risk, ${ }^{22}$ so the reduced parasympathetic modulation found in the present study, observed through lower values of the RMSSD,
pNN50, SD1, and HF ms ${ }^{2}$ indices in the cancer group, may indicate higher cardiovascular risk and a worse prognosis in this population.

High HRV is associated with higher levels of parasympathetic modulation and longer survival in patients with myocardial infarction and those under palliative care. ${ }^{21}$ Regarding cancer, alterations in autonomic modulation influence the development and prognosis of the disease.

In a study carried out with an animal model it was shown that stimulation of the nervous system with the release of catecholamines can activate beta-adrenergic receptors in tumour cells, leading to more aggressive growth and dissemination of malignant cells, whereas in humans the use of beta- has been shown to be beneficial in reducing the risk of recurrence in patients with breast cancer. ${ }^{23}$ Regarding parasympathetic modulation, Erin et al. ${ }^{24}$ demonstrated that vagal blockade promotes the increase of metastases in mice. 


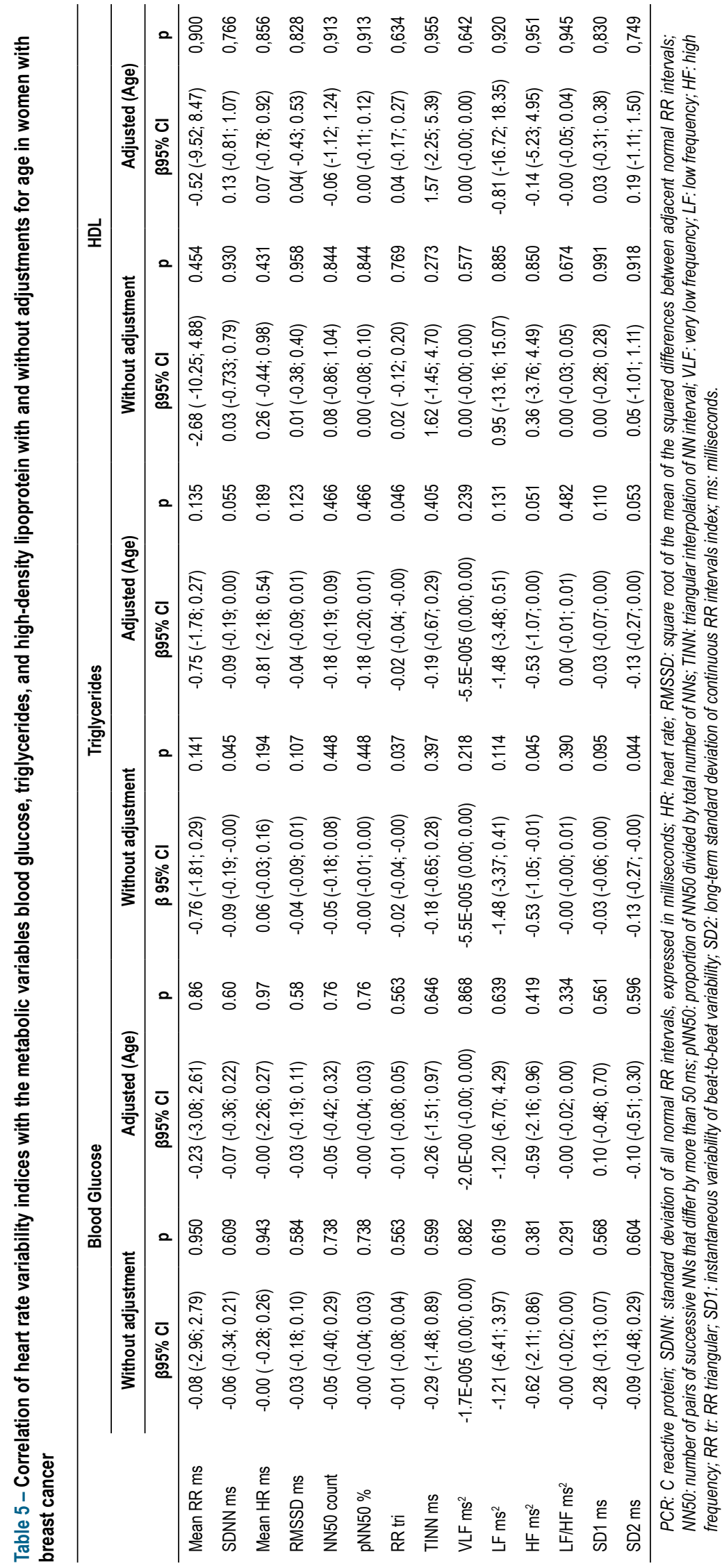


Estrogen is indicated as the main cardioprotective factor for women and its reduction in menopause is related to the increase in sympathetic activity; a circumstance that may be aggravated in women with breast cancer due to treatment of the disease with Al. ${ }^{19}$ In this context, the present study presents important results regarding the clinical framework of these patients.

Regarding the correlation between HRV indices and cardiovascular biochemical variables, no correlation was observed with CRP, fasting glycemia, or HDL-Cholesterol. CRP has been highlighted, since elevated levels of this protein are associated with an increased risk of peripheral arterial disease, myocardial infarction, stroke, and sudden death, ${ }^{25}$ and high levels of CRP are predictive of poor prognosis for cancer patients, regardless of lifestyle, menopausal status, and the presence of CVD. ${ }^{12}$

Although no statistically significant negative correlations were observed between HRV and CRP levels, indices reflecting overall variability and parasympathetic modulation presented marginal statistical values in relation to the correlation with CRP levels of the cancer group (SDNN $-\beta 95 \% \mathrm{Cl}=0.92$, $\mathrm{p}=0.082 ; \mathrm{SD} 2-\beta 95 \% \mathrm{Cl}=1.26, \mathrm{p}=0.136 ; \mathrm{RMSSD}-\beta$ $95 \% \mathrm{Cl}=-0.50,=0.089 ; \mathrm{SD} 1-\beta 95 \% \mathrm{Cl}=0.36, \mathrm{p}=0.092)$.

Regarding the correlation between HRV and the triglyceride indices, we observed an inversely proportional correlation of triglycerides with the $\mathrm{HFms}{ }^{2}$ index $\beta 95 \% \mathrm{Cl}=-0.53$; $p=0.045)$ and, although not significant, the SD1 index presented similar behavior $(\beta 95 \% \mathrm{Cl}=-0.03$; $p=0.095)$. In addition, the indices that reflect global variability, SDNN and SD2, also presented inversely proportional correlations with the triglycerides $(S D N N-\beta 95 \% \mathrm{Cl}=-0.09 ; \mathrm{p}=0.045$; $\mathrm{SD} 2-\beta 95 \% \mathrm{Cl}=-0.13 ; \mathrm{p}=0.044)$, suggesting that changes in autonomic modulation in women with breast cancer, characterized by a reduction in HRV, may be associated with a worse lipid profile. Studies show that ANS imbalance is associated with hormonal and metabolic alterations since this system plays a fundamental role in metabolic control. ${ }^{26}$

The study presents some methodological points to be raised. In the analyzes, we did not take into account associated heart diseases or the use of medications which could alter cardiac activity. In addition, the sample size considered for correlation analyzes may not have been sufficient for correlations between CRP, blood glucose, HDL-Cholesterol and HRV to be considered statistically significant.

Despite this, the study presents an important clinical implication regarding alterations in the autonomic modulation of women with breast cancer who use $\mathrm{Al}$ and the inversely proportional relationship between HRV and triglyceride values in this population, suggesting a higher risk of CVD and a worse prognosis for these women. This information reinforces the need to use preventive strategies that are safe and effective in the clinical approach of these patients.

\section{Conclusion}

Based on our findings, women with breast cancer who use $\mathrm{Al}$ present reductions in HRV compared to women without cancer and in these women HRV indices are inversely correlated with triglyceride values.

\section{Author contributions}

Conception and design of the research: Paulo TRS, Viezel J, Freitas Jr. IF; acquisition of data: Paulo TRS, Viezel J; analysis and interpretation of the data: Gonzaga, LA, Vanzella LM; statistical analysis: Gonzaga, LA, Vanzella LM, Vanderlei LCM; writing of the manuscript: Gonzaga, LA, Paulo TRS, Vanzella LM; critical revision of the manuscript for intellectual content: Freitas Jr. IF, Vanderlei LCM.

\section{Potential Conflict of Interest}

No potential conflict of interest relevant to this article was reported.

\section{Sources of Funding}

There were no external funding sources for this study.

\section{Study Association}

This article is part of the thesis of Doctoral submitted by Thais Reis Silva de Paulo, from Programa de Pós graduação em Ciências da Motricidade - UNESP.

\section{Ethics approval and consent to participate}

This study was approved by the Ethics Committee of the Faculdade de Ciências e Tecnologia - UNESP under the protocol number 672.7715.1.00005402/2015. All the procedures in this study were in accordance with the 1975 Helsinki Declaration, updated in 2013. Informed consent was obtained from all participants included in the study.

\section{References}

1. Jemal A, Bray F, Center MM, Ferlay J, Ward E, Forman D. Global cancer statistics. CA Cancer J Clin. 2011;61(2):69-90.

2. World Health Organization. Breast cancer: prevention and control [Internet] Geneva: World Health Organization; 2018 [citado nov. 2018]. Disponível em: http://www.who.int/cancer/detection/breastcancer/en/

3. Vance V, Mourtzakis M, McCargar L, Hanning R. Weight gain in breast cancer survivors: prevalence, pattern and health consequences. Obes Rev. 2011;12(4):282-94
4. Gao Q, Patani N, Dunbier AK, Ghazoui Z, Zvelebil M, Martin LA, et al. Effect of aromatase inhibition on functional gene modules in estrogen receptorpositive breast cancer and their relationship with antiproliferative response. Clin Cancer Res. 2014;20(9):2485-94.

5. Mao H, Bao T, Shen X, Li Q, Seluzicki C, Im EO, et al. Prevalence and risk factors for fatigue among breast cancer survivors on aromatase inhibitors. Eur J Cancer. 2018 Sep;101:47-54.

6. Mendelsohn ME. Protective effects of estrogen on the cardiovascular system Am J Cardiol. 2002;89(12A):12E-17. 


\section{Original Article}

7. Abderrahman B, Jordan VC. Successful Targeted Therapies for Breast Cancer: the Worcester Foundation and Future Opportunities in Women's Health. Endocrinology. 2018;159(8):2980-90.

8. Caro-Morán E, Fernàndez-Lao C, Galiano-Castillo N, Cantarero-Villanueva I, Arroyo-Morales M, Dìaz-Rodríguez L. Heart Rate Variability in Breast Cancer Survivors After the First Year of Treatments : A Case-Controlled Study. Biol Res Nurs. 2016;18(1):43-9.

9. Lakoski SG, Jones LW, Krone RJ, Stein PK, Scott JM. Autonomic dysfunction in early breast cancer: incidence, clinical importance, and underlying mechanisms. Am Heart J. 2015;170(2):231-41.

10. Nathan L, Shi W, Dinh H, Mukherjee TK, Wang X, Lusis AJ, et al. Testosterone inhibits early atherogenesis by conversion to estradiol: Critical role of aromatase. PNAS. 2001;98(6):3589-93.

11. Mouridsen H, Keshaviah A, Coates AS, Rabaglio M, Castiglione-Gertsch M, Sun Z, et al. Cardiovascular Adverse Events During Adjuvant Endocrine Therapy for Early Breast Cancer Using Letrozole or Tamoxifen : safety analysis of BIG 1-98 trial. J Clin Oncol. 2007;25(36):5715-22.

12. Allin $\mathrm{KH}$, Nordestgaard $\mathrm{BG}$, Flyger $\mathrm{H}$, Bojesen SE. Elevated pre-treatment levels of plasma $\mathrm{C}$-reactive protein are associated with poor prognosis after breast cancer : a cohort study. Breast Cancer Res. 2011;13(3):R55.

13. Pierce BL, Ballard-Barbash R, Bernstein L, Baumgartner RN, Neuhouser $\mathrm{ML}$, Wener $\mathrm{MH}$, et al. Elevated biomarkers of inflammation are associated with reduced survival among breast cancer patients. J Clin Oncol. 2009;27(21):3437-44.

14. American Cancer Society. Understanding a Breast Cancer Diagnosis Types of Breast Cancer [Internet]. Atlanta: American Cancer Society; 2018 [citado nov. 2018]. https://www.cancer.org/content/dam/CRC/PDF/ Public/8580.00.pdf.

15. Barbosa MP, Silva NT, Azevedo FM, Pastre CM, Vanderlei LC. Comparison of Polar ${ }^{\circledR}$ RS800G $3^{\text {TM }}$ heart rate monitor with Polar ${ }^{\circledR}$ S $810 \mathrm{i}^{\mathrm{TM}}$ and electrocardiogram to obtain the series of RR intervals and analysis of heart rate variability at rest. Clin Physiol Funct Imaging. 2016;36(2):112-7.

16. Tarvainen MP, Niskanen JP, Lipponen JA, Ranta-Aho PO, Karjalainen PA. Kubios HRV -- heart rate variability analysis software. Comput Methods Programs Biomed. 2014;113(1):210-20.
17. Task Force of The European Society of Cardiology and The North American Society of Pacing and Electrophysiology. Heart rate variability: standards of measurement, physiological interpretation, and clinical use. Eur Heart J. 1996;17(3):354-81.

18. Vanderlei LC, Pastre CM, Hoshi RA, Carvalho TD, Godoy MF. Basic notions of heart rate variability and its clinical applicability. Rev Bras Cir Cardiovasc. 2009;24(2):205-17.

19. Neves VFC, Perpetuo NM, Sakabe DI, Catai AM, Gallo Jr L, Silva de Sá MF, et al. Analysis of spectral indexes for heart rate variability in middle-aged men and postmenopausal women. Rev Bras Fisioter. 2006;10(4):401-6.

20. Friedenreich CM, Neilson HK, Woolcott CG, Wang Q, Stanczyk FZ, Mctiernan A, et al. Inflammatory marker changes in a yearlong randomized exercise intervention trial among postmenopausal women. Cancer Prev Res. 2012;5(1):98-108

21. Giese-Davis J, Wilhelm FH, Tamagawa R, Palesh O, Neri E, Taylor CB, et al. Higher vagal activity as related to survival in patients with advanced breast cancer: an analysis of autonomic dysregulation. Psychosom Med. 2017;77(4):346-55.

22. Thayer JF, Lane RD. The role of vagal function in the risk for cardiovascular disease and mortality. Biol Psychol. 2007;74(2):224-42.

23. Saarto T, Sievanen H, P Kellokumpu-Lehtinen P, Nikander R, Vehmanen L, Huovinen R, et al. Effect of supervised and home exercise training on bone mineral density among breast cancer patients. A 12-month randomised controlled trial. 2012;23(5)1601-12.

24. Erin N, Akdas BG, Harms JF, Clawson GA. Vagotomy enhances experimental metastases of 4THMpc breast cancer cells and alters substance P level. Regul Pept. 2008;151(1-3):35-42.

25. Greenland P, Alpert JS, Beller GA, Benjamin EJ, Budoff MJ, Fayad ZA, et al. 2010 ACCF / AHA Guideline for Assessment of Cardiovascular Risk in Asymptomatic Adults: a report of the American College of Cardiology Foundation/American Heart Association Task Force on Practice Guidelines. J Am Coll Cardiol. 2018;56(25):e50-103.

26. Rinaldi W, Ribeiro TAS, Marques AS, Fabricio GS, Tofolo LP, Gomes RM, et al. Effect of small litter size on the autonomic and metabolic responses of Wistars rats. Rev. Nutr. 2012;25(3):321-30. 